\title{
An Approximate Analytical Solution of Van Genuchten Model
}

\author{
Liang $\mathrm{Mi}^{1}$, a, Wei Luo ${ }^{2, b}$ \\ ${ }^{1}$ China Merchants Chongqing Communications Technology Research \& Design Institute CO.,LTD, \\ Chongqing 400067, China \\ ${ }^{2}$ College of Hydraulic \& Environmental Engineering, Three Gorges University, Yichang 443002, \\ China \\ aoilmar@126.com, bwluo@Lzu.edu.cn
}

Keywords: approximate analytical solution, Richards' equation, van Genuchten model.

Abstract. It is analyzed the wetting front position and derived an approximate analytical solution of Richards' equation (RE) with van Genuchten model, which is usually used to determine the soil and water characteristic curve in porous medium. Based on Boltzmann transformation, a special variable is constructed for RE approximation. In the end, an example is shown to confirm the accuracy of present method.

\section{Introduction}

Richards' equation (RE) is a standard, frequently used approach to predict water movement in variably saturated media. Usually, $\mathrm{RE}$ is a non-linear partial differential equation (PDE) of saturation and can be expressed as ${ }^{[1]}$

$$
\frac{\partial S}{\partial t}=\frac{\partial}{\partial x}\left(D(S) \frac{\partial S}{\partial x}\right)
$$

where $S$ is the water saturation in unsaturated porous media, $x$ and $t$ are the space and the time coordinates, $D(S)$ is the diffusivity function of saturation. We consider the infiltration of water in a semi-infinite domain and the initial-boundary conditions are

$$
\left.S\right|_{x=0}=S_{L},\left.S\right|_{x \rightarrow+\infty}=S_{0},\left.S\right|_{t=0}=S_{0} \text {, }
$$

where $S_{L}$ and $S_{0}$ are an constant moisture contents in wetting front analysis.

In present study, much approaches such as Adomian decomposition method (ADM) ${ }^{[2]}$, homotopy perturbation method $(\mathrm{HPM})^{[3]}$, differential transform method (DTM) ${ }^{[4]}$, traveling wave method $(\mathrm{TWM})^{[5]}$, extend Heaslet \& Alksne technique ${ }^{[6]}$ and so on, has been done to derive the exact solution of power law and exponential law diffusivities. These techniques are very powerful in solving complex non-linear problems. Nevertheless, there are not many analytical solution in some more complex diffusivity function, for instance, van Genuchten model ${ }^{[7]}$, which is used to quantitative describe the relationship of soil and water characteristic. Influenced and motivated by research before, it is solved one-dimensional $\mathrm{RE}$ by constructing a special variable based on Boltzmann transformation, which changes RE into an ordinary differential equation (ODE). Applying term wise derivative, the ODE is approximated by a series solution. The present solution can be suitable for arbitrary diffusivity and initial parameter $S_{0}$ in water infiltration. At last, an example of van Genuchten model is used to test the accuracy of present solution.

\section{Van Genuchten Model}

In this section, we define the problem to be solved by introducing the van Genuchten models. Assuming porosity $\varphi$ being a constant, the hydraulic diffusivity $D(S)$ of van Genuchten model could be expressed as ${ }^{[8]}$

$$
D(S)=\frac{K_{s}}{\varphi} \frac{\partial H}{\partial S}
$$

where $H$ is the pressure head, $K_{s}$ is the effective hydraulic conductivity. 
In van Genuchten model, $H$ it is expressed as a function of $S$

$H=\frac{1}{\alpha}\left[\left(\frac{S-S_{r}}{S_{s}-S_{r}}\right)^{-1 / m}-1\right]^{1 / n}$,

where $S_{r}$ is the residual volumetric water content, $S_{s}$ is the saturated volumetric water content, $\alpha$ is a parameter related to the mean pore-size, $n$ is a parameter related to the uniformity of the pore-size distribution, and $m=1-1 / n$. Ks is defined as ${ }^{[8]}$

$K_{s}=\frac{k_{r} \rho g k_{s}}{\mu}$,

where $k_{s}$ is the intrinsic permeability and $\mu$ is the dynamic viscosity of water and $k_{r}$ is the relative permeability which could be expressed as a function of $S$ in van Genuchten model ${ }^{[8]}$

$k_{r}=\left(\frac{S-S_{r}}{S_{s}-S_{r}}\right)^{1 / 2}\left[1-\left(1-\left(\frac{S-S_{r}}{S_{s}-S_{r}}\right)^{1 / m}\right)^{m}\right]^{2}$.

\section{Analysis the RE}

Introducing a new saturation $S_{1}\left(=S-S_{0}\right)$, the initial condition Eq. (3) is simplified as

$\left.S_{1}\right|_{t=0}=0$,

and RE in Eq. (1) can be written as

$\frac{\partial S}{\partial t}=\frac{\partial}{\partial x}\left(D\left(S_{1}+S_{0}\right) \frac{\partial S_{1}}{\partial x}\right)$.

The boundary conditions Eq. (2) are changed into

$\left.S_{1}\right|_{x=0}=S_{L}-S_{0},\left.S_{1}\right|_{x \rightarrow+\infty}=0$.

Introduced the Boltzmann variable $\phi=x / \sqrt{t}$, and Eq. (9) is transformed as ${ }^{[1]}$

$-\frac{1}{2} \phi \frac{\mathrm{d} S_{1}}{\mathrm{~d} \phi}=\frac{\mathrm{d}}{\mathrm{d} \phi}\left(D\left(S_{1}+S_{0}\right) \frac{\mathrm{d} S_{1}}{\mathrm{~d} \phi}\right)$

Then the initial condition Eq. (8) and boundary conditions Eq. (10) are

$\left.\phi\right|_{S_{1}=0} \rightarrow+\infty,\left.\phi\right|_{S_{1}=S_{L}-S_{0}}=0$.

Thus RE (1) with boundary conditions Eq. (2) and initial condition Eq. (3) are changed into ODE Eq. (11) with boundary conditions Eq. (12).

Integrating on Eq. (11), we obtain

$\frac{\mathrm{d} \phi}{\mathrm{d} S_{1}} \int_{0}^{S_{1}} \phi \mathrm{d} S_{1}=-2 D\left(S_{1}+S_{0}\right)$

the boundary conditions remain to be Eq. (12). Here, we construct a special variable ${ }^{[9]}$

$\xi=\int_{S_{1}}^{S_{L}-S_{0}} \frac{D\left(S_{1}+S_{0}\right)}{S_{1}} \mathrm{~d} S_{1}$,

and $n_{1}(=1,2,3, \ldots)$ order approximate solution of $\phi$ in Eq. (13) can be expressed as ${ }^{[9]}$

$\phi \approx \sum_{i=1}^{n} U_{i}\left(\int_{S_{1}}^{S_{L}-S_{0}} \frac{D\left(S_{1}+S_{0}\right)}{S_{1}} \mathrm{~d} S_{1}\right)^{i}$,

which is satisfied the boundary conditions Eq. (12). In Eq. (15), $n$ and $U_{i}$ are parameters. Applying term wise derivative approach to Eq. (13), we have

$\left.\frac{\mathrm{d}^{(k)}}{\mathrm{d} S_{1}^{(k)}}\left(\frac{\mathrm{d} \phi}{\mathrm{d} S_{1}} \int_{0}^{S_{1}} \phi \mathrm{d} S_{1}\right)\right|_{S_{1}=S_{L}-S_{0}}=-\left.2 \frac{\mathrm{d}^{(k)} D\left(S_{1}+S_{0}\right)}{\mathrm{d} S_{1}^{(k)}}\right|_{S_{1}=S_{L}-S_{0}}$, 
where $k=0,1,2,3, \ldots, n-1$. Substituting Eq. (15) in Eq. (16) and solving a non-linear algebraic equations, the parameter $U_{i}$ can be obtained.

\section{Numerical Simulation}

In this section, an example of van Genuchten model is given to confirm the accuracy of present method. The parameters are shown in Table $1^{[8]}$.

Table 1: Parameters of van Genuchten model

\begin{tabular}{|c|c|c|c|}
\hline Symbol & Parameter & Numerical & Unit \\
\hline$\varphi$ & Porosity & 0.33 & {$[-]$} \\
\hline$k_{s}$ & Intrinsic permeability & $2.95 \mathrm{E}-13$ & {$\left[\mathrm{~m}^{2}\right]$} \\
\hline$S_{r}$ & $\begin{array}{l}\text { Residual volumetric water } \\
\text { content } \\
\text { Saturation volumetric water }\end{array}$ & 0 & {$[-]$} \\
\hline$S_{\max }$ & content & 1 & {$[-]$} \\
\hline$\alpha$ & van Genuchten parameters & 1.43 & {$[1 / \mathrm{m}]$} \\
\hline$n$ & van Genuchten parameters & 1.506 & {$[-]$} \\
\hline$\mu$ & Dynamic viscosity & $1 \mathrm{E}-03$ & {$\left[\mathrm{~N} \cdot \mathrm{s} / \mathrm{m}^{2}\right]$} \\
\hline
\end{tabular}

Substituting the parameters in Table 1 into Eqs. (4)-(7), we have

$D\left(S_{1}+0.303\right)=D_{0}\left(S_{1}+0.303\right)^{-3.476190476}\left[1-\left(1-\left(S_{1}+0.303\right)^{1 / 0.336}\right)^{0.336}\right]^{2}\left[\left(S_{1}+0.303\right)^{-1 / 0.336}-1\right]^{-0.336}$,

in which $D_{0}=1.21069 \mathrm{E}-05$, and $S_{1}=S-0.303$. We take $S_{L}=0.9, S_{0}=0.303$ as the values in initial and boundary conditions Eqs. (2)-(3). Applying the approach in Eqs. (13)-(16), we obtain 1-2 order approximate solutions are

1 order approximate solution

$\frac{x}{\sqrt{t}} \approx 1813.021223 \int_{S_{1}}^{0.597} \frac{D\left(S_{1}+0.303\right)}{S_{1}} \mathrm{~d} S_{1}$.

2 order approximate solution

$\frac{x}{\sqrt{t}} \approx 1923.77364 \int_{S_{1}}^{0.597} \frac{D\left(S_{1}+0.303\right)}{S_{1}} \mathrm{~d} S_{1}-309697713.60325\left(\int_{S_{1}}^{0.597} \frac{D\left(S_{1}+0.303\right)}{S_{1}} \mathrm{~d} S_{1}\right)^{2}$.

Compared with FEM, which is divided into 400 units and calculated for results with saturation $S$ as the exact solution, the relative errors of $S$ obtained from Eq. (19) are shown in table 2 and the present method shows the results of 1-2 orders approximations of Eqs. (18)-(19) in Fig. 1.

Table 2: Results of $S$ obtained from FEM and present method

\begin{tabular}{cccc}
\hline$\phi\left[\mathrm{mm} / \mathrm{s}^{1 / 2}\right]$ & 2 order approximate solution & FEM & $\begin{array}{c}\text { Relative error } \\
{[\%]}\end{array}$ \\
\hline 1.619250829 & 0.3031 & 0.3039 & $-0.2569 \%$ \\
1.44384539 & 0.33 & 0.334397 & $-0.4626 \%$ \\
1.42255545 & 0.35 & 0.352055 & $-0.5837 \%$ \\
1.375058227 & 0.4 & 0.400794 & $-0.1981 \%$ \\
1.282188727 & 0.5 & 0.499821 & $0.0358 \%$ \\
1.158155749 & 0.6 & 0.5994 & $0.1001 \%$ \\
0.969473347 & 0.7 & 0.6998 & $0.0286 \%$ \\
0.650541403 & 0.8 & 0.79968 & $0.0400 \%$ \\
0 & 0.9 & 0.9 & $0.0000 \%$ \\
\hline
\end{tabular}




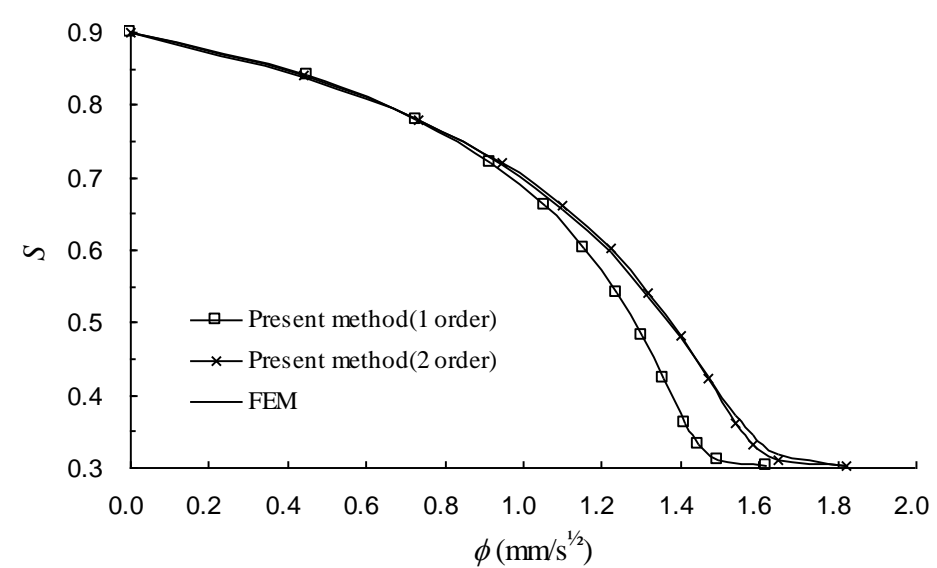

Fig. 1 Calculation results obtained from present method and FEM

In Fig. 1, it can be obtained the saturation $S$ decreases from 0.9 to 0.303 as the space coordinate $x$ increases. The 2 order approximate solution is very close to the exact solutions in Fig. 1. In Table 2, the maximum relative error value of present method is $-0.5837 \%$ in $x=1.42255545 \mathrm{~mm}$ for 2 order approximation solution.

\section{Conclusion}

In this paper, an approximation solution of RE is derived by applying Boltzmann transformation and term by term differentiation in Eq. (16). The hydraulic conductivity in RE can be arbitrary function of saturation $S$, and the initial condition parameter $S_{0}$ could be an arbitrary constant. The presented example demonstrates the accuracy of the present solution by comparing the present results with the results obtained from the FEM.

\section{Acknowledgment}

This work is supported by the National Natural Science Foundation of China (Grant no.11302119).

\section{References}

[1] M.B. Parlange, S.N. Prasad, J. Y. Parlange and M.J.M. Romkens: Water Resour. Res. Vol. 28 (1992), p. 2793

[2] N. Khodabakhshi, S.M. Vaezpour and D. Baleanu: Fract. Calc. Appl. Anal. Vol. 17 (2014), p. 382

[3] T. P. Witelski: Transport Porous Med. Vol. 27 (1997), p. 121

[4] M. Omidvar, A. Barari, M. Momeni and D. D. Ganji: Geomech. Geoeng. Int. J. Vol. 5 (2010), p. 127

[5] T. P. Witelski: Adv. Water Resour. Vol. 28 (2005), p. 1133

[6] D. Lockington, J.Y. Parlange and P. Dux: Mater. Struct. Vol. 32 (1999), p. 342

[7] M.T. van Genuchten: Soil Sci. Soc. Am. J. Vol. 44 (1980), p. 892

[8] O. Kolditz, U.J. Gorke, H. Shao and W. Wang: Thermo-Hydro-Mechanical-Chemical Processes in Porous Media (Springer-Verlag Berlin Heidelberg, Berlin 2012).

[9] X. Chen, Y. Dai: Appl. Math. Mech. Vol. 37 (2016), p. 169 